\title{
Disentangling and Lamellar Thickening of Linear Polymers during Crystallization: Simulation of Bimodal and Unimodal Molecular Weight Distribution Systems
}

Zengqiang Zhai, Claudio Fusco, Julien Morthomas, Michel Perez, Olivier Lame*

Univ de Lyon, INSA Lyon, MATEIS, UMR CNRS 5510, 69621 Villeurbanne, France

*Correspondence author. *E-mail: olivier.lame@insa-lyon.fr

\begin{abstract}
We have perform coarse-grained molecular dynamics simulations to study the isothermal crystallization of bimodal and unimodal molecular weight distribution (MWD) polymers with equivalent average molecular weight $\left(M_{w}\right)$. By using primitive path analysis we can monitor the entanglement evolution during the process of crystallization. We have discovered a quantitative correlation between the degree of disentanglement and crystallinity, indicating that chain disentanglement permits the process of crystallization. In addition, the crystalline stem length also displays a linear relation with the degree of disentanglement at different temperatures. Based on the observation in our simulations, we can build a scenario of the whole process of chain disentangling and lamellar thickening on the basis of chain sliding diffusion. Furthermore, we have enough evidence to infer that the temperature dependence of crystalline stem length is basically a result of temperature dependence of chain sliding diffusion. Our observations are also in agreement with Hikosaka's sliding diffusion theory. Compared to the unimodal system, the disentanglement degree of the bimodal system is more delayed than its crystallinity due to the slower chain sliding of long-chain component; the bimodal system reaches a larger crystalline stem length at all temperatures due to the promotion of higher chain sliding mobility of short-chain component.
\end{abstract}

Keywords: bimodal polymer; degree of disentanglement; chain sliding diffusion; lamellar thickening; coarse-grained molecular dynamics; semicrystalline polymer; 
Semicrystalline polymers with bimodal Molecular Weight Distribution (MWD) are good candidates for applications because of the improved processability along with promoted mechanical performance. ${ }^{1}$ Bimodal MWD polymers are composed of low- and high- molecular weight $\left(M_{w}\right)$ contents. Some researchers ${ }^{2-6}$ have prepared bimodal polymer blends in experiments and found that bimodal melts have promoted nucleation rate and processability of the material. In particular, the relationship between property and micro-structure of bimodal polyethylene (PE) has been studied, ${ }^{7-9}$ and it is believed that the entanglements play an essential role for the outstanding properties of bimodal PE.

Molecular Dynamics simulations have also been used to study crystallization ${ }^{10-15}$ and microstructure ${ }^{16-20}$ of semicrystaline polymers. Nevertheless, there are only few studies addressing the simulation of crystallization in relation with entanglements ${ }^{14,15,21}$ of bimodal polymers. Moyassari et al. ${ }^{14,15}$ monitored entanglement concentrations during crystallization of PE bimodal blends. Luo et al. ${ }^{10,21-23}$ studied the crystallization of unimodal PVA chains and also blends with very short chains (considered as solvent phase), and found a linear relation between crystalline stem length and entanglement length estimate. It is worth noting that these contributions all attributed the promoted nucleation rate of bimodal system to the increased proportion of long chain content, and neglected the fact that the average $M_{w}$ also increased. However, the question remains: what is the main factor for the crystallization and lamellar thickening of bimodal polymers? The increased long chain content or the increased average $M_{w}$ ? Therefore, in order to avoid the effect of $M_{w}$, we have created bimodal and unimodal systems with the equivalent average $M_{w}$.

In this work, we have studied systems of bimodal and unimodal MWD with equivalent average $M_{w}$. Coarse-grained MD simulations have been performed to investigate the isothermal crystallization behavior of these systems at various temperatures. The entanglements have been continuously monitored in the process of crystallization. The crystal growth and thickening process are described and discussed afterwards. The simulation methods and techniques for the analysis of the entanglements are presented at the end of this article. 


\section{Results \& Discussion}

\section{Isothermal crystallization}

We use a coarse-grained polymer model ${ }^{24}$ where linear polymer chains consist of "beads" representing a few structural units. The energy, length and time units are given by $\varepsilon, \sigma$ and $\tau$ respectively (with $\tau=\sqrt{m \sigma^{2} / \epsilon}, m$ is the mass unit). As we have mentioned in the introduction, a bimodal system B166 has been created with 100 long chains (chain length is 500 beads) and 500 short chains (chain length is 100 beads). To avoid an effect of average $M_{w}$, we have created a unimodal system U166 with 600 chains of length 166 beads, with the same average $M_{w}$. Please note that in this work we use an optimized weak interaction potential (see our previous article ${ }^{24}$ ), which favours homogeneous nucleation. Please refer to the Methods section at the end of this paper for the simulations details and for the methods of the post-processing analysis.

The two systems are relaxed through a long period of $5.0 \times 10^{5} \tau$ in the NPT ensemble, and the equilibrium is confirmed by the convergence of Mean Square Internal Distance (MSID) curves. Then the systems are submitted to a fast cooling process to the target temperatures $2.3,2.1,2.0$, 1.9 and $1.7 \epsilon / k_{\mathrm{B}}$ respectively. We have chosen these temperatures because the glass transition temperature is $1.39 \epsilon / k_{\mathrm{B}}$, and $2.3 \epsilon / k_{\mathrm{B}}$ is the maximum temperature at which homogeneous crystallization can be obtained within acceptable computational time. ${ }^{25}$ Afterwards, the temperatures of the systems are kept constant for the isothermal treatment, until the crystallization is adequately fulfilled. Figure 1a shows the enthalpies per bead as a function of isothermal time at different temperatures. We can see that only at temperature $2.3 \epsilon / k_{\mathrm{B}}$ the enthalpy shows an incubation time before crystallization and then starts to decrease indicating the onset of crystallization, while the crystallization occurs almost instantly at lower temperatures. The whole isothermal process lasts for $4.0 \times 10^{5} \tau$, and the enthalpy of all the systems reaches a plateau, indicating that all the systems have reached maximum crystallinities. In Figure 1(b e), we also provide four snapshots at the early stage and at the end of crystallization for system B166 at temperatures 2.3 and $1.9 \epsilon / k_{\mathrm{B}}$ respectively. At the early stage, much more nuclei have been formed at temperature of $1.9 \epsilon / k_{\mathrm{B}}$ than at high temperature of $2.3 \epsilon / k_{\mathrm{B}}$, which is consistent with experimental observations ${ }^{26,27}$ and with classical nucleation kinetics. ${ }^{28,29}$ At the end, large lamellae with tapered edge have been obtained at high temperature of $2.3 \epsilon / k_{\mathrm{B}}$ : the largest crystalline domain of system B166 has an average stem length of $22.66 \sigma$ and a maximum expansion of 84.76 
$\sigma$ perpendicular to crystal orientation, and the largest crystalline domain of system U166 has an average stem length of $22.61 \sigma$ and a maximum expansion of $91.35 \sigma$ perpendicular to crystal orientation. The ratio of lateral expansion versus the crystalline stem length is approximately four. However, for lower temperature this ratio gradually decreases: for system B166, these ratios at temperatures $2.3,2.0,1.9,1.7 \epsilon / k_{\mathrm{B}}$ are respectively $3.74,3.54,3.42,2.57$. The number of crystalline stems of the largest crystallite of system B166 at temperatures $2.3,2.0,1.9,1.7 \epsilon / k_{\mathrm{B}}$ are respectively $1432,1212,1125,270$. A larger number of crystallites is detected at lower temperature.

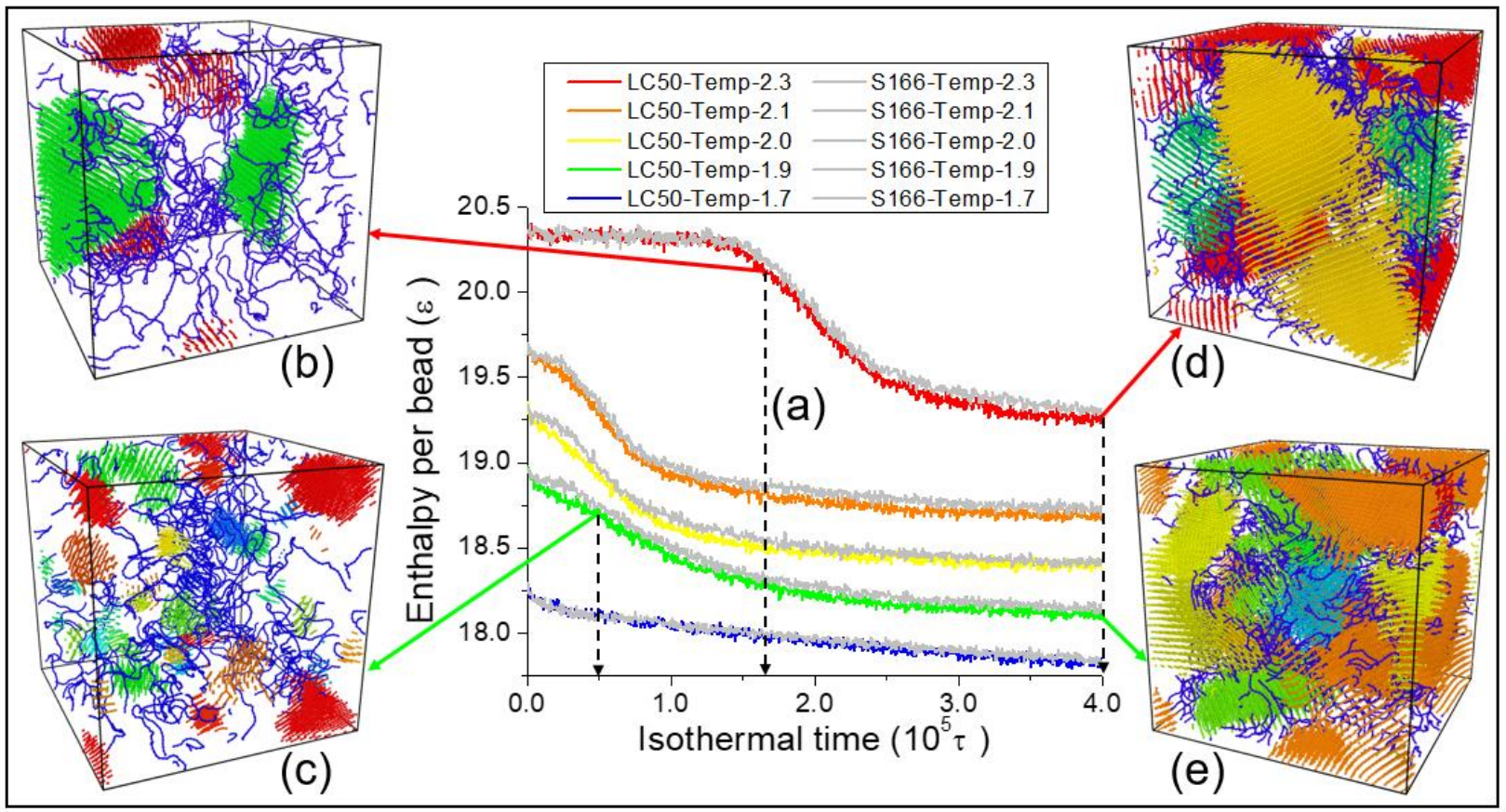

Figure 1: (a) Thermograms of isothermal treatment of systems B166 and U166, at constant temperatures 1.7 to $2.3 \epsilon / k_{\mathrm{B}}$ respectively for a period of $4.0 \times 10^{5} \tau$. The enthalpy is calculated according to $H=E+p V$. (b)(c) Snapshots of system B166 at the early stage of crystallization with isothermal temperatures of 2.3 and $1.9 \epsilon / k_{\mathrm{B}}$, respectively. (d)(e) Snapshots of system B166 at the end of isothermal treatment with isothermal temperatures of 2.3 and $1.9 \epsilon / k_{\mathrm{B}}$, respectively. The colors in snapshots $(b \sim e)$ : blue represents several complete chains traveling through amorphous and crystal phases, the other colors represent different crystallites (one color for each crystallite).

In order to quantify the crystallinity, we have used our hierarchical-clustering method. With this method, ${ }^{24,25}$ we are able to calculate the crystallinity of systems B166 and U166, as well as 
their long-chain crystallinity and short-chain crystallinity. For example, the crystallinity of long chains in B166 is defined as the number of long-chain beads in crystal phase divided by total number of long-chain beads. From Figure 2a, we can see that after an incubation time system B166 crystallizes earlier than U166 at isothermal temperature of $2.3 \epsilon / k_{\mathrm{B}}$, following a faster growth rate, and then it reaches a plateau displaying a final crystallinity slightly higher than that of U166. However, the crystallinity-time curves of B166 and U166 are only weakly different. It is the same case for the final crystallinities at all investigated temperatures (Figure 2b). It seems that the final crystallinity is determined by the average $M_{w}$ and independent of bimodality. Krumme et al. ${ }^{30,31}$ and Shen et al. ${ }^{32,33}$ have also found that the bimodal blends of PE behaved insensitive to bimodality, with respect to microscopic properties like crystallinity and density.

The crystallinities of long-chain and short-chain components of system B166 are also plotted in Figure 2a. The crystallinities of the two components show the same trend as the overall crystallinity. Short-chain crystallinity is always higher than that of long-chain component, because short chains diffuse and align faster. This indicates that short chains promote the crystallization during the crystal growth process, while long chains hinder it instead. The overall crystallinity of B166, close to that of unimodal system U166, is a compromise between that of the short and long chains. Triandafilidi et al. ${ }^{34}$ have also reported a similar conclusion in the crystallization competition of the two components. In Figure 2c, we have plotted the ratio of final long-chain crystallinity divided by short-chain crystallinity at various temperatures. This ratio decreases with temperature, indicating that the difference of crystallization rate between short chains and long chains becomes larger at high temperatures. This mainly results from the increasing difference of diffusion behavior between short and long chains and from the fact that the diffusion coefficient increases exponentially with temperature. ${ }^{35,36}$ Cosgrove et al. ${ }^{37}$ also reported that the diffusion coefficient of long chains and short chains increases with increasing temperature. 


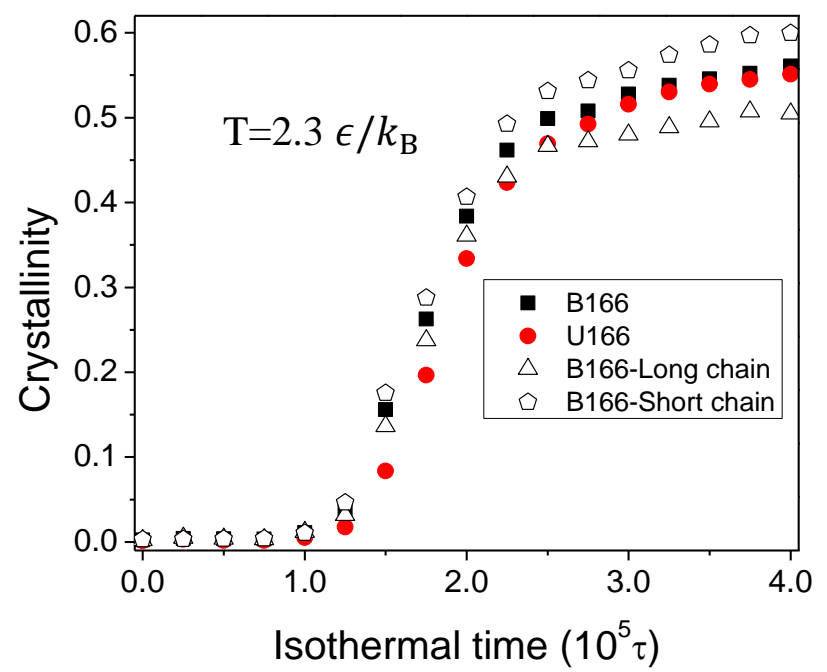

(a)

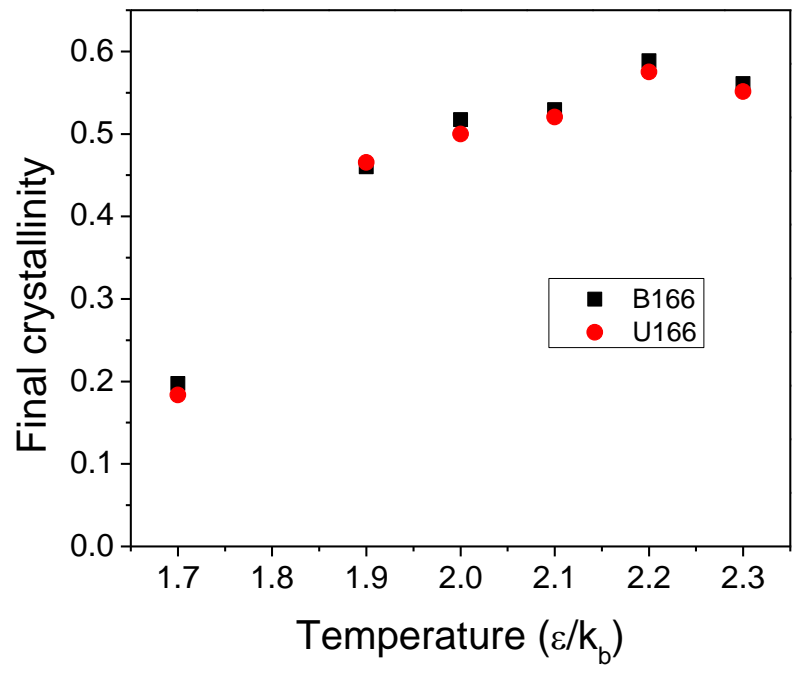

(b)

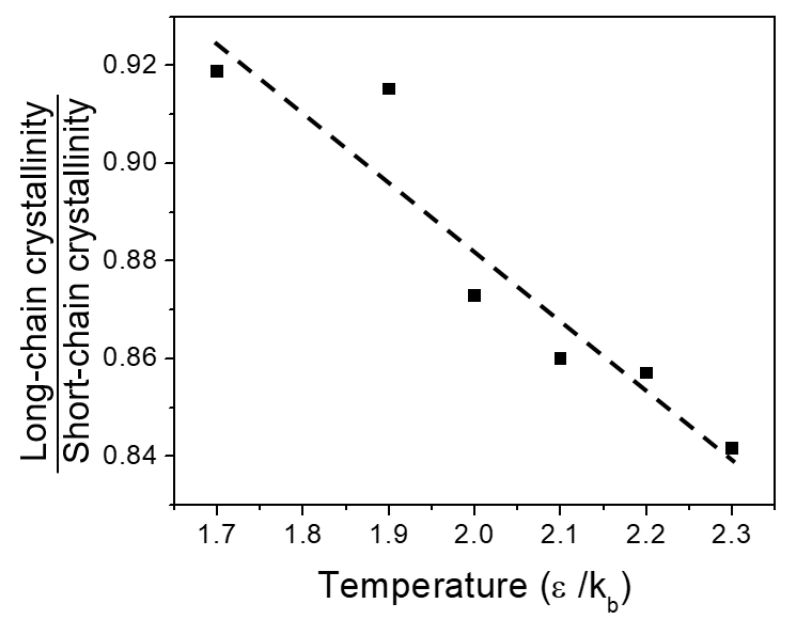

(c)

Figure 2: (a) Evolution of crystallinity of systems B166 (overall/long-chain/short-chain) and U166 at temperature $2.3 \epsilon / k_{\mathrm{B}}$, as a function of isothermal time. (b) Final crystallinity at the end of isothermal treatment of systems B166 and U166, as a function of temperature. (c) Ratio of longchain crystallinity divided by short-chain crystallinity as a function of temperature.

\section{Entanglement evolution}

As shown in some experimental studies, ${ }^{38-40}$ entanglement characteristics in the polymer melt play an important role for the crystallization selection of morphology and crystal thickening. Therefore, it is essential to trace the evolution of entanglements during the crystallization process. For easier comparison, here we use $\langle\mathrm{Z}>$ per bead as an indication of entanglement concentration, i.e. average number of entanglements per chain divided by chain length. Figure 3 a shows the entanglement concentration of systems B166 and U166 as a function of isothermal time at 
temperature $2.3 \epsilon / k_{\mathrm{B}}$. The entanglement concentrations of B166 and U166 first slightly drop at the beginning of isothermal treatment, which is considered to be the delayed reaction to the preceding fast cooling. Then the $\langle\mathrm{Z}\rangle$ per bead slowly increases during the incubation time which is caused by the increase of chain stiffness. This increase during incubation is consistent with previous simulation studies and theoretical models. ${ }^{41-43}$ Afterwards, with the onset of crystal growth, the concentration of entanglements rapidly decreases, indicating a disentanglement process. Finally the crystallization saturates and disentanglement reaches a plateau at the end of isothermal treatment. Interestingly, the $\langle\mathrm{Z}\rangle$ per bead of B166 is very similar to that of U166 during the incubation period, but at the end it drops to a lesser degree compared to U166. Clearly systems B166 and U166 hold similar crystallinities (Figure 2b) even if they disentangle differently, as it will be further discussed in this paper. Figure $3 \mathrm{a}$ also shows the disentanglement process of long/short chains in bimodal system of B166. The difference of the initial value of $\langle\mathrm{Z}\rangle$ per bead between long chains and short chain results from the effect of the $M_{w}$ and the correction term of equation (3). Long chains exhibit higher entanglement concentration than short chains, and eventually achieve different decreases of entanglements. These observations also hold for crystallization process at other temperatures, except that no evident incubation period is detected.

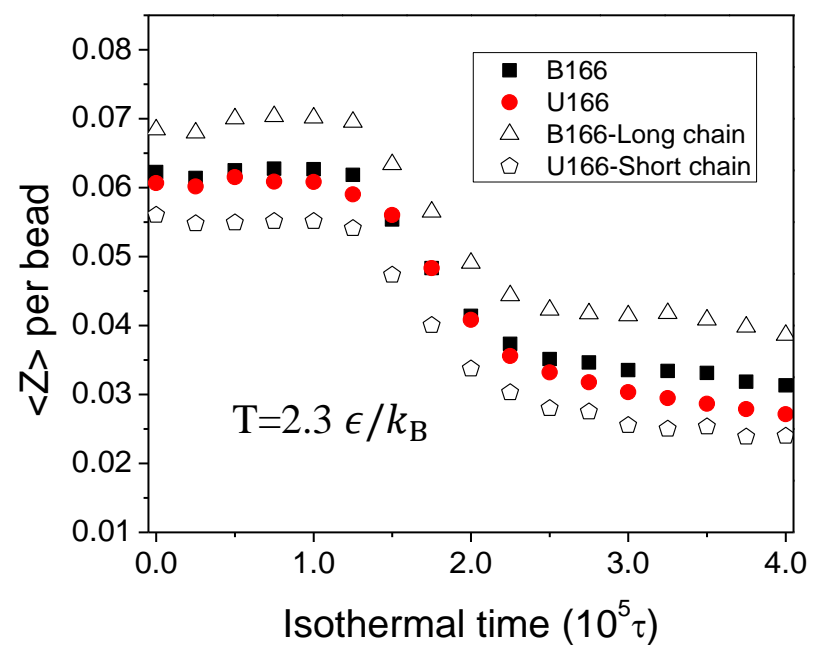

(a)

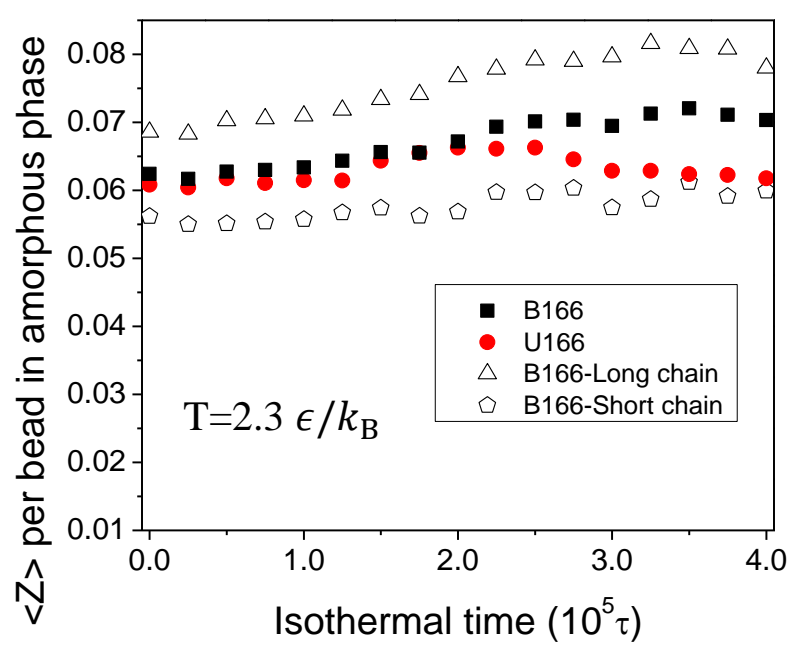

(b) 


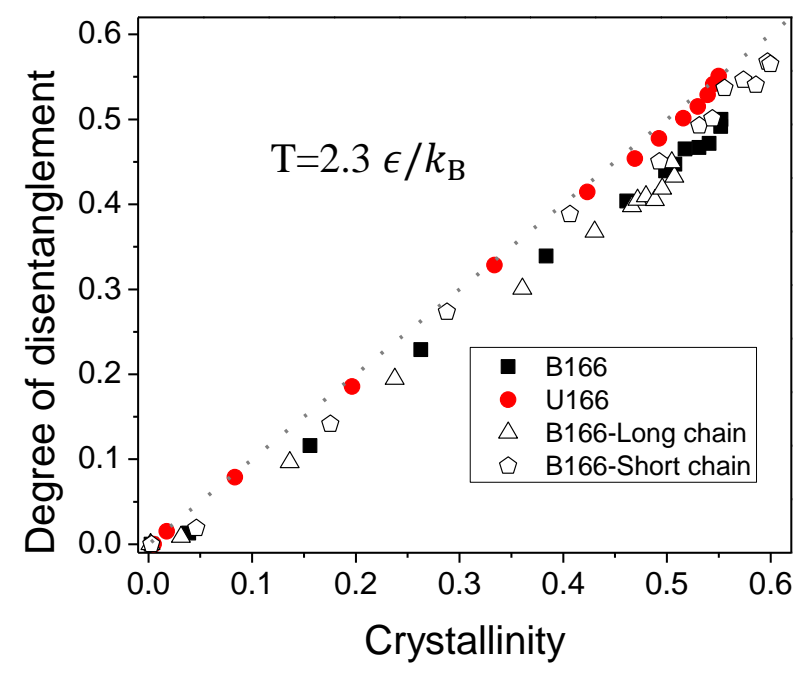

(c)

Figure 3: (a) The number of entanglements per bead as a function of isothermal time at temperature $2.3 \epsilon / k_{\mathrm{B}}$. (b) The number of entanglements per bead in amorphous phase as a function of isothermal time at temperature $2.3 \epsilon / k_{\mathrm{B}}$. (c) Degree of disentanglement as a function of crystallinity, at temperature $2.3 \epsilon / k_{\mathrm{B}}$.

The role of entanglements and possible mechanisms of disentanglement during polymer crystallization has been debated in literature, ${ }^{44-47}$ but experimental investigation on entanglement properties is difficult. ${ }^{39,45}$ No quantitative relations between the degree of entanglement and crystallization processes have been made. In order to quantify the degree of disentanglement, we define it as $\frac{<Z>\left(t_{0}\right)-<Z>(t)}{<Z>\left(t_{0}\right)}$, where $<Z>(t)$ is the entanglement concentration of the system at time $t$ of isothermal period and $t_{0}$ is the incubation time, i.e. the time when crystallization starts. Based on the calculation of incubation time in our previous work, ${ }^{25} t_{0}$ for system B166 is $1.22 \times$ $10^{5} \tau$ and for system U166 it is $1.26 \times 10^{5} \tau$. With this definition, we are able to plot the degree of disentanglement of systems B166 and U166, as well as that of long chains and short chains of B166, as a function of crystallinity (Figure 3c).

It needs to be clarified that in Figure $3 \mathrm{c}$ each component correlates with its own crystallinity. For example, the disentanglement degree of B166 is a function of average crystallinity of system B166, and that of long chains is a function of long-chain crystallinity of system B166. The dashed line is $y=x$. From Figure $3 \mathrm{c}$, we can see that the degrees of disentanglement of all the systems and components always follow their own crystallinities. Hence we conclude that the crystallization process is fundamentally a process of chain disentangling, and that the crystallinity is basically the 
degree of chain disentanglement. We have provided a quantitative correlation between crystallinity and degree of disentanglement. Prior to Luo et al. and Moyassari et al., Lee and Rutledge ${ }^{48}$ had reported a similar phenomenon in the process of mechanical deformation of simulated polyethylene. They found that the evolution of the entanglement length estimate always follows the trend of crystallinity during both slow and fast deformation process, which suggests that entanglements are created or eliminated readily in response to the production (melting) or removal (recrystallization) of amorphous material. Other researchers have also debated about the relationship between polymer crystallization and disentanglement; ${ }^{15,44,45,49,50}$ nevertheless, to the best of the authors' knowledge, we have never seen similar quantitative reports. For system U166, the entanglement degree always follows its own crystallinity. As for system B166, the average degree of disentanglement is a bit delayed with respect to crystallinity, and long-chain component is similarly delayed while the degree of disentanglement of short chains is close to its crystallinity. It seems that in system B166, long-chain component dominates the delay of disentanglement degree. Why does this delay exist in bimodal systems, and especially in the long-chain component? In order to answer this question, we monitor the degree of entanglements in the amorphous phase (Figure 3b).

As there are few entangled defects in the crystalline domain, we assume that for a specific chain the entanglements are spread along the amorphous fragment, and we assume that the length of this amorphous fragment is $N \cdot\left(1-X_{c}\right)$ ( $N$ is chain length and $X_{c}$ is crystallinity). With this assumption, we replot the entanglement concentration in the amorphous phase (defined as $<Z>_{\text {amorph }}$ ) as a function of isothermal time (Figure $3 b$ ). We can see that $<Z>_{\text {amorph }}$ per bead has an evident increase during the crystal growth for both systems B166 and U166. The difference is that U166 drops back to similar levels as before crystallization, whereas the drop of B166 is slower and still in progress at the end of isothermal. As for long chains of B166, the increase of $\langle Z\rangle_{\text {amorph }}$ is even more evident and draws back slower than U166 and the average value of B166, while $<Z>_{\text {amorph }}$ for short chains of B166 exhibits no evident increase or decrease in the whole process of crystallization. Luo et al. ${ }^{10,21,23}$ have observed that entanglements are almost preserved during crystallization in the amorphous melts in their PVA simulations. Figure $3 \mathrm{~b}$ brings to a similar conclusion, but in our case, the entanglement concentration of amorphous phase increases slightly and drops at the end. For long chains, the withdrawing is slow and long processing, as confirmed in the extended simulation of system B166. Moyassari et al. 
15,50 also reported that entanglements were less preserved for the short chains than the long chains in the process of crystallization. It seems that the delay of disentanglement degree of bimodal system B166 (compared to its crystallinity) is due to the slow reaction of long-chain component in amorphous phase. This will be further discussed in the following sub-section.

\section{Lamellar thickening}

Another fundamental question concerning polymer crystallization is the effect of bimodal MWD and temperature on crystalline stem length. In general, low crystallization temperatures lead to thinner lamellae. In Figure 4a, we show a linear relation between the final stem length and degree of disentanglement for systems B166 and U166 at temperature of $2.3 \epsilon / k_{\mathrm{B}}$. This indicates that crystal thickening is also controlled by disentanglement process of the chains. It has been indicated ${ }^{10,23}$ that the stem length selection is due to the restriction of the entanglements, and less entangled regions can crystallize faster with longer crystalline stem lengths. Luo et al. have found a linear relation between the entanglement length estimate at the beginning of crystallization and the resulting crystalline stem length. ${ }^{10,21,22}$ These works pointed out the memory effect of entanglements on final stem length, but neglected the disentangling process during crystallization. In this work, the simulation systems are submitted to a fast cooling to the desired temperatures $\left(2.3,2.1,2.0,1.9\right.$ and $\left.1.7 \epsilon / k_{\mathrm{B}}\right)$ from the equilibration temperature of $3.3 \epsilon / k_{B}$. In fact, the entanglements react relatively slowly and the entanglement state hardly evolves during the fast cooling. This suggests that the average entanglement density at the onset of crystallization for the different temperatures is almost the same for our systems. Even for bimodal system and unimodal system, the entanglement concentrations at the onset of crystallization (see Figure 3a) are very close: this cannot account for the stem length difference of the two systems. In this paper, at variance with the work of Luo et al. where non-isothermal crystallization has been performed and the effect of thermal history has been discussed, we study isothermal crystallization from the same initial entangled system. We particularly focus on the steps preceding crystallization and provide more insights into the chain disentangling and chain sliding. 


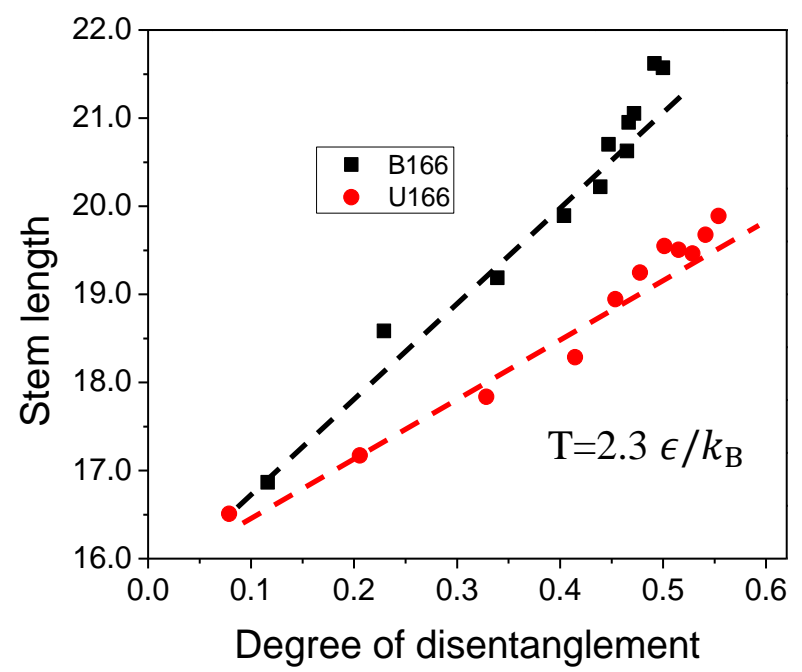

(a)

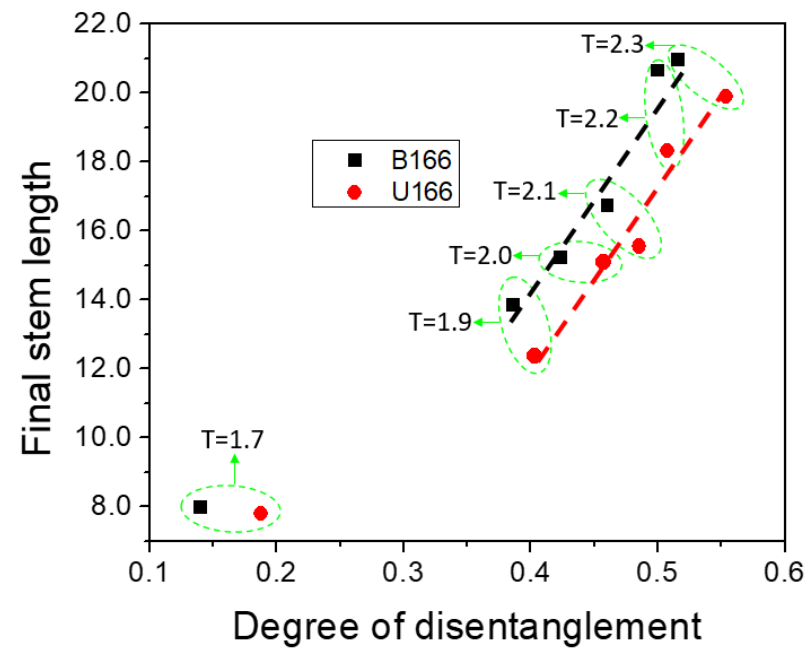

(b)

Figure 4: (a) Average crystalline stem length as a function disentanglement degree of systems B166 and U166, at temperature $2.3 \epsilon / k_{\mathrm{B}}$. (b) Average crystalline stem length at the end of crystallization for different temperatures (unit of temperature labels is $\epsilon / k_{\mathrm{B}}$ ), as a function disentanglement degree of systems B166 and U166.

Based on Figure 3 and Figure 4a, we are now able to explain the scenario of the whole process of crystallization. Crystallization is a process where polymer chains disentangle within the interface between a crystallite and the melt, and then rearrange into large crystals via chain diffusion. Apparently, there are two types of diffusion of the chains: sliding diffusion, which is diffusion of a polymer chain along its own axis, and lateral diffusion, which is the displacement of a chain fragment in the lateral direction. Luo et al. ${ }^{10}$ indicated that the process of polymer crystallization is accompanied by the sliding and folding of chain fragments, which are also the two modes of chain disentangling. Basically the chains fold to the growth front through lateral diffusion forming short crystal stems, and then the stems thicken through chain sliding. This could also be visually observed from Figure 6, in which the entanglement evolution of two individual chains of B166 (a long and a short chain) is presented. The primitive paths of the folds show that the folds are disentangled and mainly form the folded-end surface of the crystallites. As discussed in previous works, the crystallization occurs via the unentangled stems moving to the growth front through chain folding. ${ }^{23,51,52}$ Our previous article ${ }^{25}$ has pointed out that long chains favor folded chain crystallization also because the interfacial free energy of folded-end surface is much lower than that of extended-end surface. On the contrary, a fully aligned long chain exhibits such a low entropy that the probability to obtain it is extremely weak. The number of kinks (representing 
entanglements) for the two chains decreases in the process of chain folding and crystal thickening, and most of the kinks fall in the interfacial and amorphous regions indicating that no entanglements exist in the crystal phase.

In order to quantify the diffusion mobility of the chains, we have calculated the mean square displacement (MSD) of the beads, as described in Harmandaris et al. ${ }^{53}$ The MSDs of the beads for systems B166 and U166 at temperature of $2.3 \epsilon / k_{\mathrm{B}}$ are shown in Figure 5a. Clearly the average MSD of the beads is not sensitive to bimodality, while the MSD of short-chain beads is higher than long-chain beads. Figure $5 \mathrm{~b}$ shows the MSDs of the centers of mass of the chains for systems $\mathrm{B} 166$ and $\mathrm{U} 166$ at temperature of $2.3 \epsilon / k_{\mathrm{B}}$. Clearly, the average MSD of the chains of the bimodal system is larger than that of the unimodal system, and the diffusion mobility of the short-chain content is much higher than that of the long-chain content. This conclusion also holds for other temperatures.
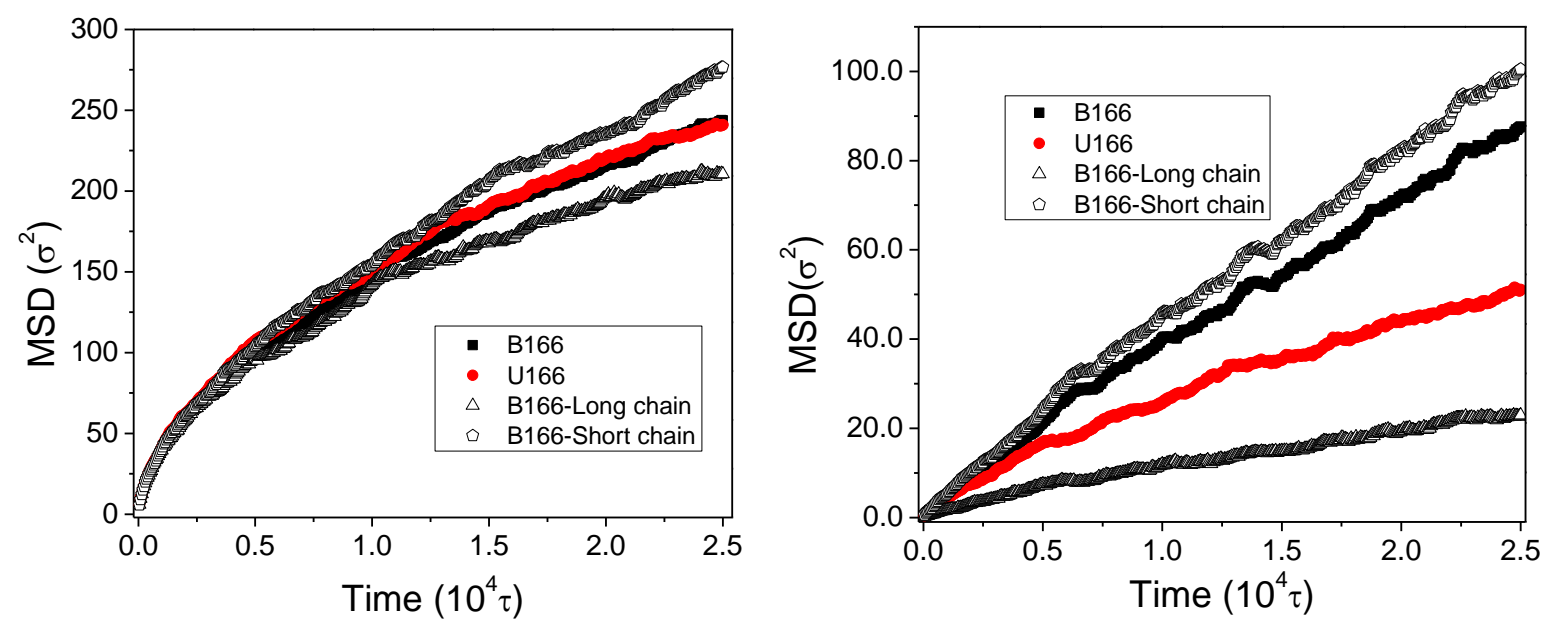

Figure 5. (a) Mean square displacement of the beads as a function of time for B166 (overall/longchain beads/short-chain beads) at temperature $2.3 \epsilon / k_{\mathrm{B}}$. (b) Mean square displacement of the center of mass of the chains as a function of time for B166 (overall/long chains/short chains) at temperature $2.3 \epsilon / k_{\mathrm{B}}$.

The short chains disentangle to a larger extent because of the high sliding mobility, in agreement with the work of Lacevic et al. ${ }^{54}$ who calculated the sliding diffusion coefficient and found that the chain sliding decreases with increasing $M_{w}$. It is worth noticing that the entanglements do not completely disappear on the crystal interface of crystallite and melt, which can be seen from the increase of the entanglement concentration in the amorphous phase (Figure 3b) especially for long chains of B166. In other words, entanglements are pushed away from the 
crystalline phase to the interface: the majority of them disappears and the rest is transmitted to the amorphous phase. This transmission is more evident for long chains because of the low chain sliding mobility. This explains the delay of disentanglement degree of long-chain component in system B166 (Figure 3c) and the fact that the stem length of B166 is larger than that of U166 (Figure 4a). In fact, due to the higher sliding mobility of short chains in B166, the stem length of bimodal system B166 is promoted by the short chains, and long-chain low mobility makes the disentanglement degree of long chains more delayed. Here we have provided insights into the disentangling and lamellar thickening process, and found evidence to support the chain sliding theory of polymer crystallization proposed by Hikosaka ${ }^{35,36}$ as well as verified its applicability to bimodal MWD polymers.

Figure 4b shows that the final stem length of system B166 and U166 at various temperatures also exhibits a linear relation with disentanglement degree. This indicates that the temperature dependence of the stem length is mainly influenced by disentanglement and bimodality. It is generally accepted that lower crystallization temperature leads to thinner lamellae, which is simply because lower temperature leads to slower chain sliding and consequently lower degree of disentanglement. This observation leads to the conclusion that the temperature dependence of the stem length can be the result of the temperature dependence of the disentangling process which is mainly controlled by chain sliding. This does not agree with the classical Lauritzen-Hoffman (LH) theory, ${ }^{28}$ which does not attribute it to sliding diffusion but is based on concepts of secondary nucleation and assumes disentanglement via reptation. The simulation model used in this work particularly promotes sliding effect as shear stress is very weak. On the other side, this conclusion is consistent with Luo et al. ${ }^{22}$ who have reported an inference that the temperature dependence of stem length can be simply the result of the entanglement length estimate, which is eventually a result of chain sliding mobility.

There are mainly two different points of view to explain the correlation between crystallization and entanglement concentration in semicrystalline polymers. In the perspective of Flory and Yoon, ${ }^{55}$ it is assumed that the polymer chains in the melt cannot disentangle because the disentangling time is longer than the inverse of the crystallization rate. In this case, the overall entanglement should be conserved in the process of crystallization and be segregated to the amorphous phase, which implies a higher entanglement concentration in the noncrystalline domain as the crystallinity increases. In the perspective of Hoffman and Miller, ${ }^{28}$ the entanglements are 
eliminated as crystallization proceeds; whether the entanglement concentration in the remaining noncrystalline fraction maintains constant or not is not clear. Based on the concept of "forced reptation", in order to have enough time to disentangle during crystallization, Hoffman argued that a chain should be extracted from its entanglements with a rate of approximately $10^{2}$ larger than the growth rate, typically applicable to nucleation regimes I and II (nucleation regimes from Hoffman theory). Investigating the transition regime of the crystal growth is totally another subject, and growth rate is involved in this work either, but the regime of nucleation can be estimated according to Hoffman theory. From Figure 5b, the average moving rate of the center of mass of the chains is approximately $3 \times 10^{-2} \sim 6 \times 10^{-2} \sigma^{2} / \tau$ at temperature $2.3 \epsilon / k_{\mathrm{B}}$, which can be considered as an indicator of the disentanglement rate. The final radius $(\sim 45 \sigma)$ of the largest crystallites of system B166 and U166 is obtained after an isothermal time of $4 \times 10^{5} \tau$, so that the growth rate is approximately $1.0 \times 10^{-4} \sigma / \tau$. Therefore, the disentanglement of the simulation systems at temperature $2.3 \epsilon / k_{\mathrm{B}}$ in this work supports Hoffman theory. A lot of experimental studies display only minor lamellar thickening with low sliding dynamics even under thermal annealing, whereas high sliding mobility is only known for polyethylene and the simple CG-MD model used in this work rather resembles polyethylene in this respect (above $\alpha_{c}$ relaxation). Based on Figure 4, we assume that the disentanglement and lamellar thickening of systems B166 and U166 in the temperature range of $1.9 \sim 2.3 \epsilon / k_{\mathrm{B}}$ verify their affiliation to nucleation regimes $\mathrm{I}$ and II. For the temperature $1.7 \epsilon / k_{\mathrm{B}}$, the stem length and degree of disentanglement are evidently much lower than at other temperatures, so it is inferred to lie in regime III. 


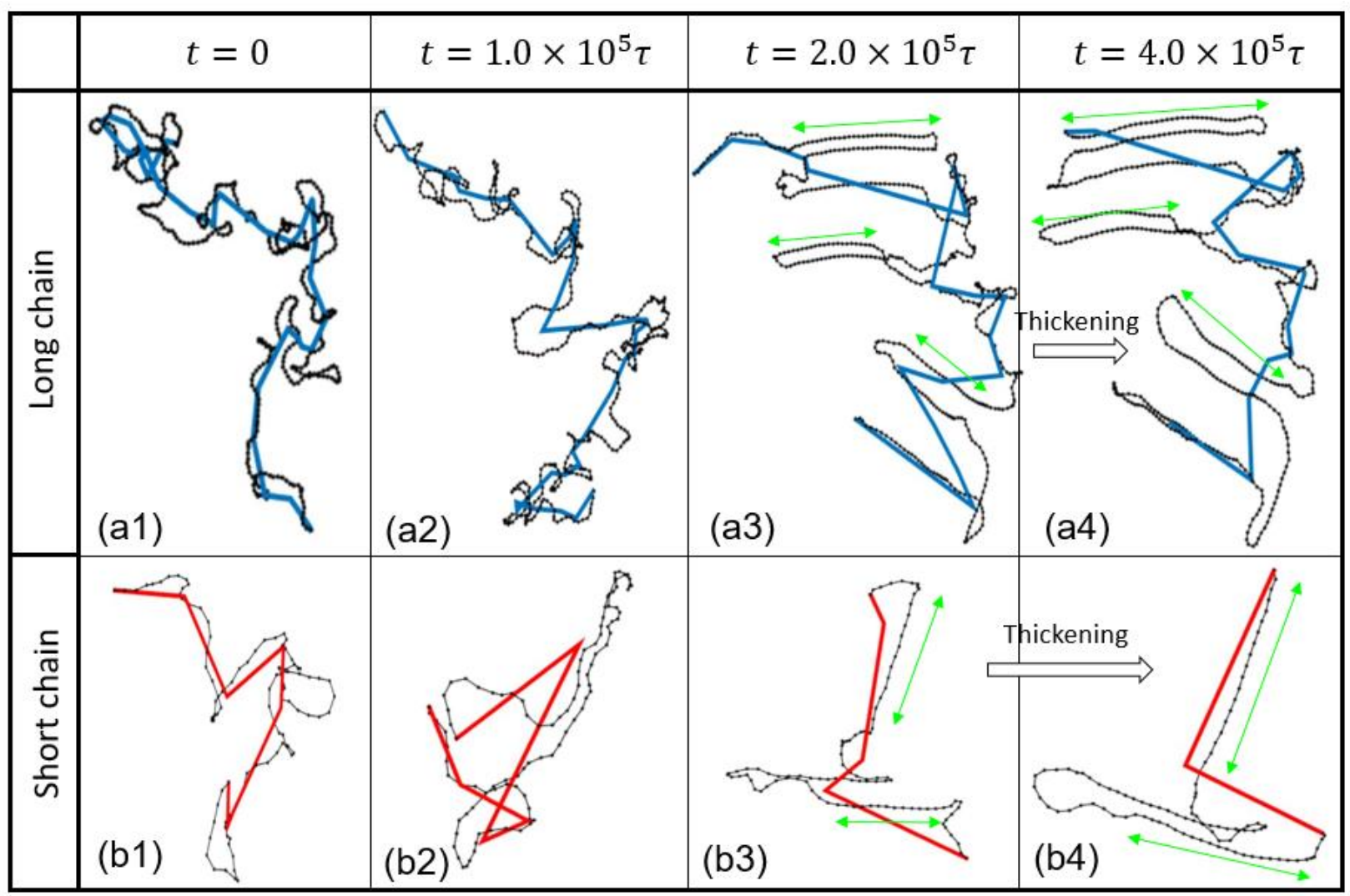

Figure 6: Configurations and Primitive Paths (PP) of two individual chains of system B166 (one is long chain, the other is short chain), during isothermal crystallization at temperature $2.3 \epsilon / k_{\mathrm{B}}$. The black thin lines are the real configurations, the red and blue thick straight lines are the Primitive Paths given by $\mathrm{Z} 1$ algorithm.

\section{Conclusions}

In this study, we have investigated the crystallization of bimodal and unimodal MWD polymers with the same average molecular weight $M_{w}$, at various temperatures. Through the primitive path analysis, the entanglement evolution has been continuously monitored during crystallization. We have shown that the crystallization process is accompanied by chain disentangling, and shown a quantitative correlation between disentanglement degree and the crystallinity. Crystallinity is a universal result of average $M_{w}$, and it is not sensitively different for bimodal and unimodal systems. By employing molecular simulations, we have described polymer crystallization as a process of chain disentangling on the interface of crystal and melted phases in the manner of chain sliding diffusion. Crystalline stem length also displays a linear relation with the degree of disentanglement at all referred temperatures, and the temperature dependence of stem length is simply a result of the temperature dependence of chain sliding diffusion. Bimodal system 
B166 exhibits a higher stem length than unimodal system U166 at all temperatures, since the low $M_{w}$ component promotes stem length because of high sliding mobility. Our observations provide direct evidence of Hikosaka's sliding diffusion theory. Observing entanglement restrictions is difficult in experiments. Our observations with MD simulation provide a quantitative analysis of the long-term debate on chain disentanglement and lamellar thickening, which is sensitive to bimodality as a result of high chain sliding mobility of the short-chain component. The important role of entanglements and chain sliding diffusion sheds a light on deeper understanding of polymer crystallization.

\section{Methods}

We use a coarse-grained polymer model ${ }^{24}$ where polymer chains consist of "beads" representing a few structural units. All simulations are performed in three dimensions using the open-source code LAMMPS. ${ }^{56}$

\section{Interaction potentials and modeling systems}

The model is based on two potentials, where energy, length and time units are given by $\varepsilon, \sigma$ and $\tau$ respectively (with $\tau_{u}=\sqrt{m \sigma^{2} / \epsilon}, m$ is the mass unit). A Finite-Extensible Non-linear Elastic (FENE) potential models intra-chain interactions of bonded beads:

$$
V_{\mathrm{FENE}}(r)=-0.5 k R_{0}^{2} \ln \left[1-\left(\frac{r}{R_{0}}\right)^{2}\right]+4 \varepsilon_{F}\left[\left(\frac{\sigma_{F}}{r}\right)^{12}-\left(\frac{\sigma_{F}}{r}\right)^{6}\right],
$$

with $k=30 \varepsilon / \sigma^{2}, R_{0}=1.5 \sigma, \varepsilon_{F}=\varepsilon$ and $\sigma_{F}=1.05 \sigma$, chosen so that unphysical bond crossing and chain breaking are avoided. Note that the value of $\sigma_{F}$ is chosen such that $V_{\mathrm{FENE}}(r=\sigma)$ is minimum. All other interactions are modelled by a simple Lennard-Jones (LJ) potential:

$$
V_{\mathrm{LJ}}(r)=4 \varepsilon_{L J}\left[\left(\frac{\sigma_{\mathrm{LJ}}}{r}\right)^{12}-\left(\frac{\sigma_{\mathrm{LJ}}}{r}\right)^{6}\right]-4 \varepsilon_{L J}\left[\left(\frac{\sigma_{\mathrm{LJ}}}{r_{c}}\right)^{12}-\left(\frac{\sigma_{\mathrm{LJ}}}{r_{c}}\right)^{6}\right],
$$

where $\varepsilon_{L J}=\varepsilon, r_{c}=5.0 \sigma$ is the cutoff radius, $\sigma_{\mathrm{LJ}}$ is an adjusted parameter of the potential that favours crystallization and crystal stability for the FENE-LJ model chosen here. We take $\sigma_{\mathrm{LJ}}=$ $1.888 \sigma$ as proposed in our previous work, ${ }^{24}$ where the polymer chains tend to align and form thermal stable crystallites. In this $\mathrm{LJ}$ potential, $\sigma_{\mathrm{LJ}}$ is approximately twice the bond length $(0.995$ $\sigma)$, resulting in the alignment of three consecutive beads for low energy purpose. In fact, the optimized LJ potential acts as an angular potential promoting the nucleation of the polymer by 
aligning the beads in the same chain. We have verified the thermodynamic stability of the optimized LJ potential and we have confirmed the homogeneous nucleation ability by isothermal crystallization treatment. By heating of a perfect crystalline configuration we have estimated the glass transition temperature and the melting temperature of the CG model with chain length of 100 beads as respectively $1.4 \epsilon / k_{B}$ and $3.1 \epsilon / k_{B}$.

In this work, we have created a bimodal system (namely B166) with 100 long chains (chain length is 500 beads) and 500 short chains (chain length is 100 beads), which makes $50 \%$ of weight fraction of long chain content. The average $M_{w}$ of system B166 is $166.7 \mathrm{~m}$. To avoid an effect of average $M_{w}$, we have created a second system (namely U166) with 600 chains of length 166 beads, with the same $M_{w}$ and total number of beads as system B166. Extensive simulations and comparisons have been done on systems B166 and U166 in this work.

To generate these systems with target number of chains and chain length, the Radical-Like Polymerization (RLP) method ${ }^{57,58}$ has been employed (see more details in Zhai et al. ${ }^{25,58}$ ). The systems are then equilibrated during $5.0 \times 10^{5} \tau$ at temperature $T=3.3 \epsilon / k_{B}$ and pressure $P=$ $0.5 \epsilon / \sigma^{3}$ in the NPT ensemble. The Nosé-Hoover thermostat and barostat are used to maintain the temperature and pressure. Newton's equations of motion are integrated with velocity-Verlet method with a time step of $0.005 \tau$. The box dimensions of the equilibrated systems of B166 and U166 at temperature $3.3 \epsilon / k_{B}$ are $71.02 \times 71.02 \times 71.02 \sigma^{3}$ and $70.94 \times 70.94 \times 70.94 \sigma^{3}$. Mean Square Internal Distance (MSID) is calculated to verify that all systems are well equilibrated. Then the systems are submitted to a fast cooling process (cooling rate: $10^{-5} \epsilon / k_{B} / \tau$ ) to the desired crystallization temperature, followed by an isothermal treatment for a long period of $4.0 \times 10^{5} \tau$ until the crystallization of each system saturates. We have selected temperatures of 2.3, 2.1, 2.0, 1.9 and $1.7 \epsilon / k_{\mathrm{B}}$, because the glass transition temperature is $1.39 \epsilon / k_{\mathrm{B}}$, and $2.3 \epsilon / k_{\mathrm{B}}$ is the maximum temperature at which homogeneous crystallization can be obtained within acceptable computational time. $^{25}$

\section{Crystallinity and entanglement analysis}

In order to quantify the crystallinity and detect the crystallites and their sizes, we use an algorithm based on hierarchical clustering. ${ }^{24,25}$. This algorithm seeks to build a hierarchy of crystal clusters and the beads belonging to the same cluster would be detected and integrated. It employs a bottom-up strategy: each bead starts its own cluster and all the clusters sharing the same bond 
orientation (within a tolerance angle of $5^{\circ}$ ) are merged as one moves up the tree structure. The crystallinity is defined as the ratio of the number of beads belonging to crystalline phase over the total number of beads. In this way, we are able to detect all crystal clusters (i.e. crystallites), and to trace all the chains passing through the crystalline phase and amorphous phase. For further details of this algorithm, one can refer to our previous works. ${ }^{24,25}$

For semicrystalline systems, the entanglement analysis methods that use pure geometrical criteria to examine the primitive path network from a polymer system, is more efficient. In this work, we use the $\mathrm{Z} 1$ algorithm ${ }^{59}$ to analyze the entanglements. The $\mathrm{Z} 1$ code extracts the primitive path of a given trajectory configuration, and determines several entanglement properties, of which we are mainly interested in $Z$, the number of entanglements (number of interior kinks) in each polymer chain, and $N_{e, k i n k}$, the entanglement length estimate. As the conformations of polymer chains no longer obey Gaussian statistics in melts near crystallization, we use the directly measured number of beads in a straight primitive path segment between two adjacent kinks as the entanglement length estimate. The corresponding estimators operating on the number of kinks are usually denoted as "classical kinks", ${ }^{59,60}$ which follow the form:

$$
\begin{aligned}
& Z=\frac{N}{N_{e, \mathrm{kink}}}-\frac{N}{N-1} \\
& N_{e, \mathrm{kink}}=\frac{N(N-1)}{Z(N-1)+N}
\end{aligned}
$$

where $N$ is the chain length (i.e. number of beads).

\section{Author information}

Corresponding author

*E-mail: olivier.lame@insa-lyon.fr

\section{Acknowledgments}

All the simulations were performed on the massively parallel computer P2CHPD of FLMSN (Fédération Lyonnaise de Modélisation et Sciences Numériques). The Chinese Scholarship Council is acknowledged for the grant of a doctoral fellowship to Zengqiang ZHAI.

\section{References}

(1) Nele, M.; Pinto, J. C. Molecular-Weight Multimodality of Multiple Flory Distributions. 
Macromol. Theory Simulations 2002, 11, 293-307.

(2) Song, S. J.; Wu, P. Y.; Ye, M. X.; Feng, J. C.; Yang, Y. L. Effect of Small Amount of Ultra High Molecular Weight Component on the Crystallization Behaviors of Bimodal High Density Polyethylene. Polymer (Guildf). 2008, 49, 2964-2973.

(3) Maus, A.; Saalwächter, K. Crystallization Kinetics of Poly(Dimethylsiloxane) MolecularWeight Blends - Correlation with Local Chain Order in the Melt? Macromol. Chem. Phys. 2007, 208, 2066-2075.

(4) Kornfield, J. A.; Kumaraswamy, G.; Issaian, A. M. Recent Advances in Understanding Flow Effects on Polymer Crystallization. Ind. Eng. Chem. Res. 2002, 41, 6383-6392.

(5) Wu, T.; Yu, L.; Cao, Y.; Yang, F.; Xiang, M. Effect of Molecular Weight Distribution on Rheological, Crystallization and Mechanical Properties of Polyethylene-100 Pipe Resins. $J$. Polym. Res. 2013, 20, 271.

(6) Sun, X.; Shen, H. W.; Xie, B. H.; Yang, W.; Yang, M. B. Fracture Behavior of Bimodal Polyethylene: Effect of Molecular Weight Distribution Characteristics. Polymer (Guildf). 2011, 52, 564-570.

(7) García, R. A.; Carrero, A.; Martín, C.; Domínguez, C. Effects of the Structural Components on Slow Crack Growth Process in Polyethylene Blends. Composition Intervals Prediction for Pipe Applications. J. Appl. Polym. Sci. 2011, 121, 3269-3276.

(8) Hubert, L.; David, L.; Séguéla, R.; Vigier, G.; Degoulet, C.; Germain, Y. Physical and Mechanical Properties of Polyethylene for Pipes in Relation to Molecular Architecture. I. Microstructure and Crystallisation Kinetics. Polymer (Guildf). 2001, 42, 8425-8434.

(9) DesLauriers, P. J.; Cole, N.; Lamborn, M. C.; White, C. G.; Lavine, B. K. Chemometric Methods for Estimating the Strain Hardening Modulus in Polyethylene Resins. Appl. Spectrosc. 2018, 72, 463-475.

(10) Luo, C.; Sommer, J. U. Frozen Topology: Entanglements Control Nucleation and Crystallization in Polymers. Phys. Rev. Lett. 2014, 112, 195702.

(11) Ko, M. J.; Waheed, N.; Lavine, M. S.; Rutledge, G. C. Characterization of Polyethylene Crystallization from an Oriented Melt by Molecular Dynamics Simulation. J. Chem. Phys. 2004, 121, 2823-2832.

(12) Yi, P.; Locker, C. R.; Rutledge, G. C. Molecular Dynamics Simulation of Homogeneous Crystal Nucleation in Polyethylene. Macromolecules 2013, 46, 4723-4733. 
(13) Ramos, J.; Vega, J. F.; Martínez-Salazar, J. Molecular Dynamics Simulations for the Description of Experimental Molecular Conformation, Melt Dynamics, and Phase Transitions in Polyethylene. Macromolecules 2015, 48, 5016-5027.

(14) Moyassari, A.; Mostafavi, H.; Gkourmpis, T.; Hedenqvist, M. S.; Gedde, U. W.; Nilsson, F. Simulation of Semi-Crystalline Polyethylene: Effect of Short-Chain Branching on Tie Chains and Trapped Entanglements. Polymer (Guildf). 2015, 72, 177-184.

(15) Moyassari, A.; Gkourmpis, T.; Hedenqvist, M. S.; Gedde, U. W. Molecular Dynamics Simulations of Short-Chain Branched Bimodal Polyethylene: Topological Characteristics and Mechanical Behavior. Macromolecules 2019, 52, 807-818.

(16) Yeh, I. C.; Lenhart, J. L.; Rutledge, G. C.; Andzelm, J. W. Molecular Dynamics Simulation of the Effects of Layer Thickness and Chain Tilt on Tensile Deformation Mechanisms of Semicrystalline Polyethylene. Macromolecules 2017, 50, 1700-1712.

(17) Men, Y.; Rieger, J.; Strobl, G. Role of the Entangled Amorphous Network in Tensile Deformation of Semicrystalline Polymers. Phys. Rev. Lett. 2003, 91, 095502.

(18) Yeh, I.-C.; Andzelm, J. W.; Rutledge, G. C. Mechanical and Structural Characterization of Semicrystalline Polyethylene under Tensile Deformation by Molecular Dynamics Simulations. Macromolecules 2015, 48, 4228-4239.

(19) Jabbari-Farouji, S.; Rottler, J.; Lame, O.; Makke, A.; Perez, M.; Barrat, J.-L. Correlation of Structure and Mechanical Response in Solid-like Polymers. J. Phys. Condens. Matter 2015, $27,194131$.

(20) Higuchi, Y.; Kubo, M. Deformation and Fracture Processes of a Lamellar Structure in Polyethylene at the Molecular Level by a Coarse-Grained Molecular Dynamics Simulation. Macromolecules 2017, 50, 3690-3702.

(21) Luo, C.; Kröger, M.; Sommer, J. U. Entanglements and Crystallization of Concentrated Polymer Solutions: Molecular Dynamics Simulations. Macromolecules 2016, 49, 90179025.

(22) Luo, C.; Sommer, J. U. Role of Thermal History and Entanglement Related Thickness Selection in Polymer Crystallization. ACS Macro Lett. 2016, 5, 30-34.

(23) Luo, C.; Sommer, J. U. Disentanglement of Linear Polymer Chains toward Unentangled Crystals. ACS Macro Lett. 2013, 2, 31-34.

(24) Morthomas, J.; Fusco, C.; Zhai, Z.; Lame, O.; Perez, M. Crystallization of Finite-Extensible 
Nonlinear Elastic Lennard-Jones Coarse-Grained Polymers. Phys. Rev. E 2017, 96, 52502.

(25) Zhai, Z.; Morthomas, J.; Fusco, C.; Perez, M.; Lame, O. Crystallization and Molecular Topology of Linear Semicrystalline Polymers: Simulation of Uni- and Bimodal Molecular Weight Distribution Systems. Macromolecules 2019, 52, 4196-4208.

(26) Miyamoto, Y.; Tanzawa, Y.; Miyaji, H.; Kiho, H. Concentration Dependence of Lamellar Thickness of Isotactic Polystyrene at High Supercoolings. J. Phys. Soc. Japan 2005, 58, $1879-1882$.

(27) Hu, W. The Physics of Polymer Chain-Folding. Phys. Rep. 2018, 747, 1-50.

(28) Hoffman, J. D.; Miller, R. L. Kinetics of Crystallization from the Melt and Chain Folding in Polyethylene Fractions Revisited: Theory and Experiment. Polymer (Guildf). 1997, 38, 3151-3212.

(29) Jabbari-Farouji, S.; Rottler, J.; Lame, O.; Makke, A.; Perez, M.; Barrat, J.-L. Plastic Deformation Mechanisms of Semicrystalline and Amorphous Polymers. ACS Macro Lett. 2015, 4, 147-150.

(30) Krumme, A.; Lehtinen, A.; Viikna, A. Crystallisation Behaviour of High Density Polyethylene Blends with Bimodal Molar Mass Distribution 2. Non-Isothermal Crystallisation. Eur. Polym. J. 2004, 40, 371-378.

(31) Krumme, A.; Lehtinen, A.; Viikna, A. Crystallisation Behaviour of High Density Polyethylene Blends with Bimodal Molar Mass Distribution 1. Basic Characteristics and Isothermal Crystallisation. Eur. Polym. J. 2004, 40, 359-369.

(32) Shen, H. W.; Xie, B. H.; Yang, W.; Yang, M. B. Thermal and Rheological Properties of Polyethylene Blends with Bimodal Molecular Weight Distribution. J. Appl. Polym. Sci. 2013, 129, 2145-2151.

(33) Shen, H. W.; Xie, B. H.; Yang, W.; Yang, M. B. Non-Isothermal Crystallization of Polyethylene Blends with Bimodal Molecular Weight Distribution. Polym. Test. 2013, 32, $1385-1391$.

(34) Triandafilidi, V.; Rottler, J.; Hatzikiriakos, S. G. Molecular Dynamics Simulations of Monodisperse/Bidisperse Polymer Melt Crystallization. J. Polym. Sci. Part B Polym. Phys. 2016, 54, 2318-2326.

(35) Hikosaka, M. Unified Theory of Nucleation of Folded-Chain Crystals and Extended-Chain Crystals of Linear-Chain Polymers. Polymer (Guildf). 1987, 28, 1257-1264. 
(36) Hikosaka, M. Unified Theory of Nucleation of Folded-Chain Crystals (FCCs) and Extended-Chain Crystals (ECCs) of Linear-Chain Polymers: 2. Origin of FCC and ECC. Polymer (Guildf). 1990, 31, 458-468.

(37) Cosgrove, T. Diffusion in Bimodal and Polydisperse Polymer Systems: 1. Bimodal Solutions of Protonated and Deuterated Polymers. Polymer (Guildf). 2002, 36, 3335-3342.

(38) Hikosaka, M.; Amano, K.; Rastogi, S.; Keller, A. Lamellar Thickening Growth of an Extended Chain Single Crystal of Polyethylene. 1. Pointers to a New Crystallization Mechanism of Polymers. Macromolecules 2002, 30, 2067-2074.

(39) Lippits, D. R.; Rastogi, S.; Talebi, S.; Bailly, C. Formation of Entanglements in Initially Disentangled Polymer Melts. Macromolecules 2006, 39, 8882-8885.

(40) Lippits, D. R.; Rastogi, S.; Höhne, G. W. H.; Mezari, B.; Magusin, P. C. M. M. Heterogeneous Distribution of Entanglements in the Polymer Melt and Its Influence on Crystallization. Macromolecules 2007, 40, 1004-1010.

(41) Foteinopoulou, K.; Karayiannis, N. C.; Mavrantzas, V. G.; Kröger, M. Primitive Path Identification and Entanglement Statistics in Polymer Melts: Results from Direct Topological Analysis on Atomistic Polyethylene Models. Macromolecules 2006, 39, 42074216.

(42) Sukumaran, S. K.; Grest, G. S.; Kremer, K.; Everaers, R. Identifying the Primitive Path Mesh in Entangled Polymer Liquids. J. Polym. Sci. Part B Polym. Phys. 2005, 43, 917-933.

(43) Hoy, R. S.; Robbins, M. O. Strain Hardening of Polymer Glasses: Effect of Entanglement Density, Temperature, and Rate. J. Polym. Sci. Part B Polym. Phys. 2006, 44, 3487-3500.

(44) Hoffman, J. D. Theoretical Aspects of Polymer Crystallization with Chain Folds: Bulk Polymers. Polym. Eng. Sci. 1964, 4, 315-362.

(45) Rastogi, S.; Lippits, D. R.; Peters, G. W. M.; Graf, R.; Yao, Y.; Spiess, H. W. Heterogeneity in Polymer Melts from Melting of Polymer Crystals. Nat. Mater. 2005, 4, 635-641.

(46) Qin, H.; Li, F.; Wang, D.; Lin, H.; Jin, J. Organized Molecular Interface-Induced Noncrystallizable Polymer Ultrathin Nanosheets with Ordered Chain Alignment. ACS Nano 2016, 10, 948-956.

(47) Keshavarz, M.; Engelkamp, H.; Xu, J.; Braeken, E.; Otten, M. B. J.; Uji-I, H.; Schwartz, E.; Koepf, M.; Vananroye, A.; Vermant, J.; Nolte, R. J.M.; De Schryver, F.; Maan, J. C.; Hofkens, J.; Christianen, P. C.M.; Rowan, A. E. Nanoscale Study of Polymer Dynamics. 
ACS Nano 2016, 10, 1434-1441.

(48) Lee, S.; Rutledge, G. C. Plastic Deformation of Semicrystalline Polyethylene by Molecular Simulation. Macromolecules 2011, 44, 3096-3108.

(49) Verho, T.; Paajanen, A.; Vaari, J.; Laukkanen, A. Crystal Growth in Polyethylene by Molecular Dynamics: The Crystal Edge and Lamellar Thickness. Macromolecules 2018, 51, $4865-4873$.

(50) Moyassari, A.; Gkourmpis, T.; Hedenqvist, M. S.; Gedde, U. W. Molecular Dynamics Simulation of Linear Polyethylene Blends: Effect of Molar Mass Bimodality on Topological Characteristics and Mechanical Behavior. Polymer (Guildf). 2019, 161, 139150.

(51) Müller-Plathe, F. Formation of Chain-Folded Structures in Supercooled Polymer Melts Examined by MD Simulations. Macromolecules 2002, 35, 1241-1252.

(52) Hu, W.; Frenkel, D.; Mathot, V. B. F. Intramolecular Nucleation Model for Polymer Crystallization. Macromolecules 2003, 36, 8178-8183.

(53) Harmandaris, V. A.; Mavrantzas, V. G.; Theodorou, D. N. Atomistic Molecular Dynamics Simulation of Polydisperse Linear Polyethylene Melts. Macromolecules 1998, 31, 79347943.

(54) Lacevic, N.; Fried, L. E.; Gee, R. H. Heterogeneous Directional Mobility in the Early Stages of Polymer Crystallization. J. Chem. Phys. 2008, 128, 014903.

(55) Flory, P. J.; Yoon, D. Y. Molecular Morphology in Semicrystalline Polymers. Nature 1978, 272, 226-229.

(56) Plimpton, S. Fast Parallel Algorithms for Short-Range Molecular Dynamics. J. Comput. Phys. 1995, 117, 1-19.

(57) Perez, M.; Lame, O.; Leonforte, F.; Barrat, J.-L. Polymer Chain Generation for CoarseGrained Models Using Radical-like Polymerization. J. Chem. Phys. 2008, 128, 234904.

(58) Mahaud, M.; Zhai, Z.; Perez, M.; Lame, O.; Fusco, C.; Chazeau, L.; Makke, A.; Marque, G.; Morthomas, J. Polymer Chain Generation for Coarse-Grained Models Using RadicalLike Polymerization. Commun. Comput. Phys. 2018, 24, 885-898.

(59) Kröger, M. Shortest Multiple Disconnected Path for the Analysis of Entanglements in Twoand Three-Dimensional Polymeric Systems. Comput. Phys. Commun. 2005, 168, 209-232.

(60) Hoy, R. S.; Foteinopoulou, K.; Kröger, M. Topological Analysis of Polymeric Melts: Chain- 
Length Effects and Fast-Converging Estimators for Entanglement Length. Phys. Rev. E 2009, 80, 031803. 
Disentangling and Lamellar Thickening of Linear Polymers during Crystallization: Simulation of Bimodal and Unimodal Molecular Weight Distribution Systems

Zengqiang Zhai, Claudio Fusco, Julien Morthomas, Michel Perez, Olivier Lame*

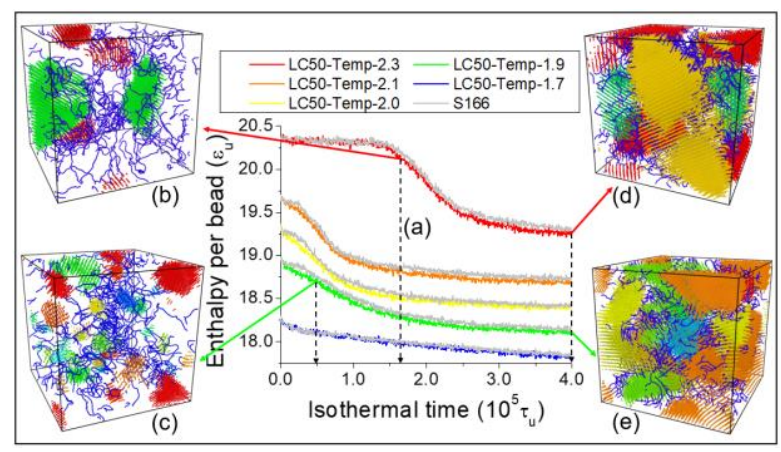

